\title{
Cambio en las redes sociales de adultos mayores beneficiarios de programas de vivienda social en Chile ${ }^{1}$
}

\author{
Reinaldo Rioseco H., ${ }^{2}$ Margarita Quezada V., ${ }^{3}$ María Elena Ducci V. ${ }^{4}$ \\ y Marisa Torres $\mathrm{H}^{5}$
}

Forma de citar

Rioseco HR, Quezada VM, Ducci VME, Torres HM. Cambio en las redes sociales de adultos mayores beneficiarios de programas de vivienda social en Chile. Rev Panam Salud Pública. 2008;23(3):147-53.

RESUMEN Objetivo. Identificar, comparar y analizar las redes sociales de dos grupos de adultos mayores pobres, beneficiarios de dos tipos de viviendas sociales, en términos de tipos de nodos, frecuencia y motivo del vínculo, antes y después del traslado a sus nuevos domicilios.

Método. Se realizó un estudio descriptivo de beneficiarios de viviendas en edificio, o bien de viviendas en condominios. El universo en estudio fue el colectivo de personas asignatarias a viviendas en edificio $(\mathrm{n}=152)$ y a viviendas en condominio $(\mathrm{n}=124)$, entre 1998 y 2001, a las cuales se les aplicó una encuesta en su domicilio.

Resultados. La mayoría de los participantes en el estudio informaron que la asignación de la vivienda implicó para ellos mudarse a barrios distantes de su antiguo domicilio, lo que provocó, entre otras consecuencias, un cambio en sus redes sociales. Ambos grupos tienen, en general, redes sociales similares, constituyendo los hijos el principal nodo de apoyo, antes y después del cambio de domicilio. Después del traslado se redujeron los vínculos en general, excepto en el caso de los adultos de vivienda en edificio, quienes incrementaron levemente sus vinculos con los hijos y en forma destacada con personas con quienes tienen vínculos débiles y que no son propiamente familiares ni amigos. Los vínculos con las instituciones también disminuyeron, aunque en el caso de las instituciones de adultos mayores aumentaron, para los residentes en condominios de $28 \%$ a $65 \%$, y para los asignatarios de edificios, de $31 \%$ a $45 \%$.

Conclusiones. Se sugiere que en los programas semejantes al estudiado se tome en cuenta la importancia que revisten la conservación y el fortalecimiento de las redes sociales creadas antes del traslado, y que se fomente la generación de vínculos en el nuevo sitio de residencia.

Palabras clave Adulto mayor, conducta de ayuda, vivienda social, pobreza, Chile.

1 Este artículo deriva de la investigación “La calidad de vida de adultos mayores que viven en vivienda básica y vivienda en condominio: un análisis comparativo", Proyecto Fondecyt $\mathrm{N}^{\circ}$ 1.040.806; 2004.

2 Instituto de Geografía, Facultad de Historia, Geografía y Ciencia Política, Pontificia Universidad Católica de Chile. La correspondencia deberá enviarse a Reinaldo Rioseco, Pontificia Universidad Católica, Instituto de Geografía, Avenida Vicuña Mackenna 4860, Santiago de Chile, Chile. T.:56 02 3544727. F.: 5602 5526028. Correo electrónico rrioseco@puc.cl
El adulto mayor (AM) debe enfrentarse al debilitamiento o pérdida de sus redes sociales con otras personas e ins-

3 Escuela de Trabajo Social, Facultad de Ciencias Sociales, Pontificia Universidad Católica de Chile.

4 Instituto de Estudios Urbanos, Facultad de Arquitectura y Urbanismo, Pontificia Universidad Católica de Chile.

5 Facultad de Medicina, Pontificia Universidad Católica de Chile. tituciones, y a la disminución de su participación social (1). Las pérdidas propias de esta etapa de la vida están más acentuadas en ambientes o estratos de pobreza $(2,3)$. En la medida en que avanza su edad, el AM se vuelve más vulnerable a cualquier circunstancia extraordinaria, tanto en el ámbito biológico como en el afectivo y el social 
(4); la salud, aunada a la situación económica, parece convertirse en una preocupación fundamental en la vejez (5).

Uno de los aspectos más importantes de la calidad de vida del AM pobre se vincula con la vivienda. Por una parte, está el hecho de que en la mayoría de los casos la familia no le ofrece espacio de acogida $y$, por la otra, en caso de alojarlo a menudo las relaciones familiares se tornan conflictivas.

Se ha entendido por redes sociales al "conjunto de relaciones interpersonales que integran a una persona con su entorno social y le permiten mantener o mejorar su bienestar material, físico y emocional" (6). Estas redes tienen especial importancia en los AM ya que, a las pérdidas ya mencionadas, hay que agregar el aumento de la dependencia respecto de personas e instituciones (7). Las redes sociales funcionan como estrategias de intercambio de apoyos formales e informales, y pueden variar en el tiempo y en el espacio, cobrando especial importancia cuando hay escasez de recursos, o bien existen riesgos o limitaciones; es ahí donde se convierten en una estrategia de supervivencia esencial (8).

Según Guzmán (9), se reconocen cuatro categorías o tipos de transferencia o de apoyos: materiales, instrumentales, emocionales y cognitivos. Desde el punto de vista de las fuentes de apoyo, se pueden distinguir las formales, que corresponden a organizaciones con objetivos explícitos y que cuentan con profesionales o voluntarios para desarrollar su labor. Por otra parte, están las fuentes informales, constituidas por personas y por grupos comunitarios no estructurados como programas de apoyo.

El Ministerio de la Vivienda y Urbanismo de Chile ha respondido a la necesidad habitacional del AM en situación de pobreza, a través del Programa de Vivienda Básica para Adultos Mayores (PVBAM), el cual considera viviendas básicas en edificio (departamentos) y viviendas en condominio, todas de carácter social, en zonas urbanas de la Región Metropolitana de Santiago. Ambas modalidades son parte de conjuntos habitacionales correspondientes a sectores de pobreza y otor- gan vivienda en calidad de comodato o arriendo, de muy bajo monto, de acuerdo con el ingreso de la persona. Los AM pueden residir en las mismas solos o acompañados de su pareja, de un hijo o de un pariente cercano, aunque en general viven solos; la mayoría han sido trasladados a comunas distantes de las de origen, lo cual llega a alterar sus redes sociales y sus desplazamientos temporales

En este estudio se analiza cómo el traslado definitivo de AM a viviendas ubicadas en comunas diferentes y distantes con respecto a las de origen, provoca alteraciones en las redes de apoyo. El objetivo principal de la investigación es identificar y analizar las redes sociales (actuales y pasadas) de los AM beneficiarios del PVBAM. Como objetivo secundario se abordarán los tipos de ayuda recibida y otorgada por los AM.

\section{MATERIALES Y MÉTODOS}

El diseño del estudio fue descriptivo transversal. El instrumento utilizado fue una encuesta aplicada en forma simultánea a los participantes y mediante la cual se consultó sobre redes y tipo de ayuda.

\section{Viviendas}

En esta investigación se consideraron los residentes de las viviendas básicas en edificio y de aquellas en condominio. En el caso de las primeras, se trata de departamentos ubicados en el primer piso (planta baja) de edificios de programas sociales, donde los AM deben convivir con un grupo heterogéneo de familias que se encuentran en otra etapa de su ciclo vital familiar, pero con características similares desde el punto de vista económico. El conjunto de AM residentes en viviendas en edificio no establece relaciones entre sí, pues sus departamentos se encuentran distribuidos en forma dispersa y no están identificados. Tampoco cuentan con una organización que les permita entablar contacto grupal con instituciones formales.
Las viviendas en condominios forman conjuntos de 20 a 25 casas de un piso, con una reja o pared alrededor que las separa del barrio circundante. En estas comunidades, las viviendas son otorgadas sólo a AM, quienes se organizan entre sí y administran lugares comunitarios tales como cocina y comedor. En algunos condominios los AM establecen vínculos formales con instituciones de la comuna (municipalidad, iglesia, etcétera).

Las viviendas están ubicadas en siete comunas de la Región Metropolitana de Santiago, de las cuales cinco, distantes del centro de la ciudad, forman parte de la planta urbana de la ciudad: Quilicura, Cerrillos, Maipú, La Pintana y Puente Alto. La parte urbana de las otras dos comunas, Colina y Buin, está alejada de la ciudad de Santiago (figura 1 ). De las viviendas, $23,9 \%$ se concentran en la comuna de Quilicura y 26,8\% en la de Cerrillos. En promedio, los AM se habían trasladado a su nueva vivienda desde hacía cuatro años.

\section{Población estudiada}

El estudio se realizó en AM de más de 60 años de edad, beneficiarios de cualquiera de los dos tipos de vivienda del PVBAM, entre 1998, año en que comenzó la asignación de viviendas en condominio, y 2001. El Servicio de Vivienda y Urbanismo del Ministerio facilitó el listado de los 373 AM asignatarios, de los cuales 155 obtuvieron una vivienda en condominio y 218 una básica en edificio.

\section{Instrumento}

El equipo investigador visitó ambos tipos de vivienda y tomó contacto con los AM. Posteriormente se diseñó y aplicó una encuesta a todos los beneficiarios en sus domicilios (octubrediciembre 2004), para lo cual se capacitó a un equipo de encuestadoras con experiencia en trabajo de campo. Se realizó una prueba piloto, se hicieron correcciones a la encuesta y después se aplicó el instrumento a la población en estudio. La encuesta incluyó variables 
FIGURA 1. Área de estudio con viviendas sociales para adultos mayores, Santiago, Chile, 2004-2005

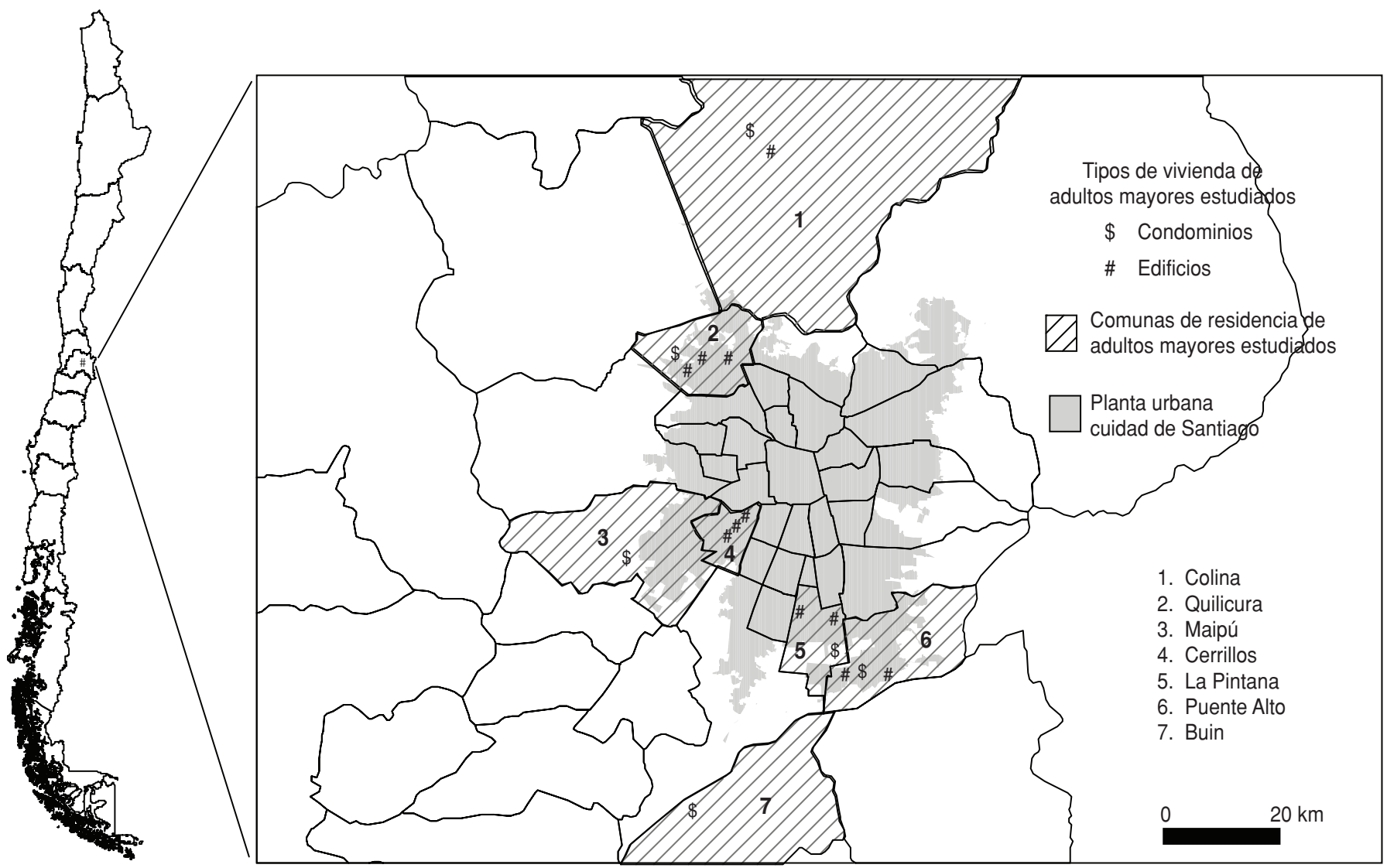

Fuente: elaboración propia.

demográficas, redes sociales (tipo) y ayuda (tipo, origen, frecuencia, beneficiarios). Se preguntó sobre las redes presentes en el momento de la aplicación de la encuesta y sobre las existentes antes del traslado de domicilio.

\section{Análisis de la información}

El equipo de investigadores se encargó de tabular y analizar los resultados de la encuesta, así como de categorizar las preguntas abiertas de acuerdo con el marco conceptual. Los resultados de las preguntas cerradas se analizaron estadísticamente. Los datos cuantitativos se procesaron con el software estadístico SPSS y se analizaron en términos de parámetros estadísticos descriptivos (media, frecuencia, distribución) para el conjunto de AM estudiados y para los dos grupos sepa- radamente. Para realizar la prueba de hipótesis se usaron pruebas estadísticas no paramétricas: $\chi^{2}$.

\section{RESULTADOS}

\section{Población estudiada}

De los $155 \mathrm{AM}$ en condominio se pudo encuestar a $80 \%(n=124)$, y de los $218 \mathrm{AM}$ en edificio a $70 \%(n=152)$, lo cual sumó 276 AM encuestados, o sea $74 \%$ del total de beneficiarios. Los entrevistados tenían en promedio 76 años de edad, con un rango entre 61 y 90 años en los condominios, y entre 60 y 94 años en los edificios. Mayoritariamente los asignatarios son mujeres: $70,5 \%$ en condominio y $77 \%$ en vivienda en edificio.

Cabe destacar que todos los encuestados se valían por sí mismos; este es un requisito indispensable del PVBAM. Cuando dicha situación cambia, el AM pasa a depender de los cuidados de su familia o bien es trasladado a alguna institución de salud.

\section{Redes}

Relaciones con personas antes del traslado. En general, antes de su mudanza los AM de ambos grupos tenían un perfil similar de relaciones, donde el principal nodo de la red eran los hijos. Del total de AM, 63\% tenían relaciones con los hijos, $29 \%$ con los hermanos y $30 \%$ con amigos. Se observaron diferencias en las relaciones con otros familiares: $46 \%$ de los $\mathrm{AM}$ en condominio se relacionaban con "otros familiares", cifra que en el caso de AM en departamentos ascendió a 59\%, lo cual es estadísticamente significativo $\left(\chi^{2}=4,811\right.$; 
CUADRO 1. Vínculos de los adultos mayores antes y después del cambio de domicilio, Santiago, Chile, 2005

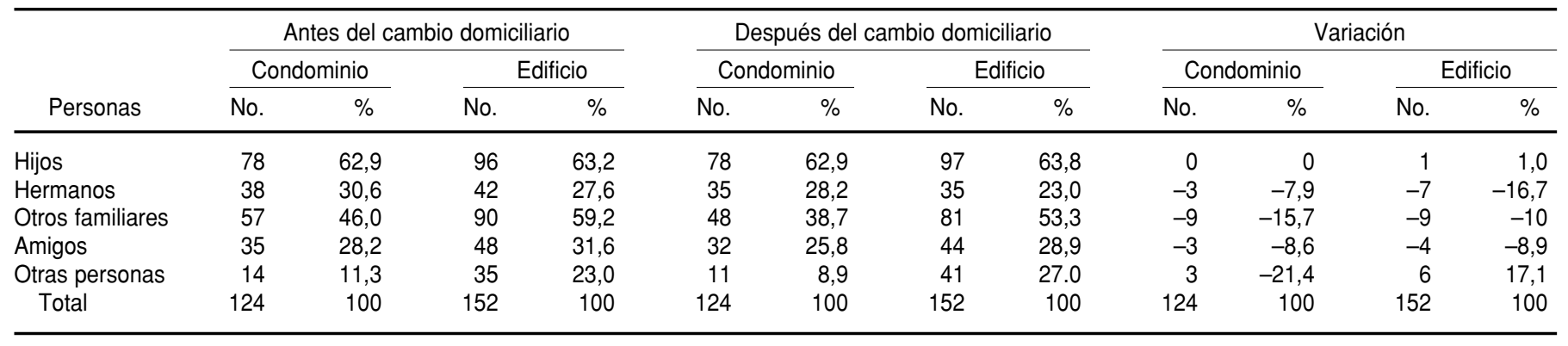

$P=0,028)$. También se observaron diferencias en el número de relaciones con "otras personas", pues en los adultos mayores en condominios la cifra era de $11 \%$ y en edificio de $23 \%$, lo que también es estadísticamente significativo $\left(\chi^{2}=6,44 ; P=0,011\right)$ (cuadro 1 ).

Relaciones con personas después del traslado. Después del cambio de domicilio, en ambos grupos los hijos siguieron siendo el nexo principal y la frecuencia de sus vínculos mantuvo la magnitud previa al traslado de domicilio. Los vínculos con los hermanos y otros familiares, en cambio, muestran disminuciones en los dos conjuntos de AM. La mayor diferencia se presenta en los hermanos de los asignatarios de viviendas en edificio, con una reducción en los contactos de casi 17\% $(n=7)$. En el caso de otros familiares, en el grupo de asignatarios en condominio se aprecia una situación similar; la disminución es de casi 16\% $(n=9)$. No obstante, estas diferencias no resultaron estadísticamente significativas ya que el valor de $P$ fue superior a 0,2 . Lo mismo puede decirse de la vinculación con los amigos, que también muestra una disminución de aproximadamente $8 \%$ en el número de casos, cifra similar en ambos grupos de AM (cuadro 1).

Los contactos con "otras personas" muestran diferencias entre ambos grupos de AM: en los que habitan en condominios se observó una disminución de $11,3 \%$ a $8,9 \%(n=14$ a $n=11)$, y en los que residen en edificio un aumento de $23 \%$ a $27 \%(n=35$ a $n=41)$. En este tipo de vínculos se mantiene una diferencia estadísticamente significativa, e inclusive después del traslado se incre- menta su significancia $\left(\chi^{2}=14,636\right.$; $P=0,001)$ (cuadro 1). Los asignatarios de ambos tipos de vivienda refieren, en $56 \%$ de las causas, que la pérdida de visitas de familiares y amigos se debe a la lejanía de sus actuales lugares de residencia.

Relaciones con organizaciones o instituciones antes del traslado. Ambos conjuntos de AM presentan una situación similar a este respecto: las instituciones de salud concentran $87 \%$ de los vínculos, superando en cerca de 20 puntos porcentuales a las instituciones de iglesia, que se sitúan en segundo lugar con $65,9 \%$ y superan por otros 20 puntos porcentuales al municipio. Las juntas de vecinos sólo representan $15,9 \%$ de los casos. Al comparar los vínculos que tenían ambos grupos de AM con estas instituciones, se observa que la mayor diferencia se encuentra en el municipio, que alcanzó sólo cuatro puntos porcentuales, sin existir diferencias significativas $(P=0,546)$ (cuadro 2).

Relaciones con organizaciones o instituciones después del traslado. Al comparar la asistencia o pertenencia a instituciones, antes y después del cambio de domicilio, se advierten ganancias y pérdidas en la frecuencia de los vínculos, de modo similar para ambos grupos de asignatarios, pero con diferente grado de intensidad. En ambos conjuntos de AM se observa estabilización de los vínculos con instituciones de salud, disminución de los vínculos con instituciones de iglesia y municipalidad y aumento de los vínculos con junta de vecinos, todos esta- dísticamente no significativos. En cambio, hay un aumento de vinculación con instituciones de AM, tanto en condominios como en viviendas en edificio. En ambos casos los valores son estadísticamente significativos, con $\chi^{2}=34,272 ; P=<1$ por mil, para condominio, y $\chi^{2}=5,576 ; P=0,018$, para edificio (cuadro 2). Al comparar los vínculos de los dos grupos con instituciones, después del traslado, se observa que sólo es estadísticamente significativa la diferencia en relación con instituciones de AM, pues $44,7 \%$ de los que residen en edificios se relacionan con las mismas, mientras que esa cifra asciende a $65,3 \%$ en los que habitan en condominios $\left(\chi^{2}=11,65 ; P=0,001\right)$ (cuadro 2).

\section{Red de ayuda}

Origen de la ayuda. Los AM de ambos grupos tienen a los hijos como principal fuente de ayuda: $36,9 \%$ en condominios y $41,3 \%$ en edificios; en segundo término a los vecinos: $22,5 \%$ en condominios y $28,3 \%$ en edificios y, en tercer lugar, al consultorio: $10,8 \%$ en condominios y 5\% en edificios. Sólo en este último caso hay una diferencia significativa entre ambos grupos: $\chi^{2}=4,821 ; P=0,028$.

Tipo de ayuda recibida. El conjunto de AM recibe ayuda que se distribuye de la siguiente forma: categoría material (pensión, remedio, dinero, comida, subsidio, ropa, alojamiento): casi $47,3 \%$ del total; categoría emocional (compañía, escucha, afecto, consuelo): $40,3 \%$; ayuda de tipo cognitivo (infor- 
CUADRO 2. Vínculo de los adultos mayores con instituciones/organizaciones antes y después del cambio de domicilio, Santiago, Chile, 2005

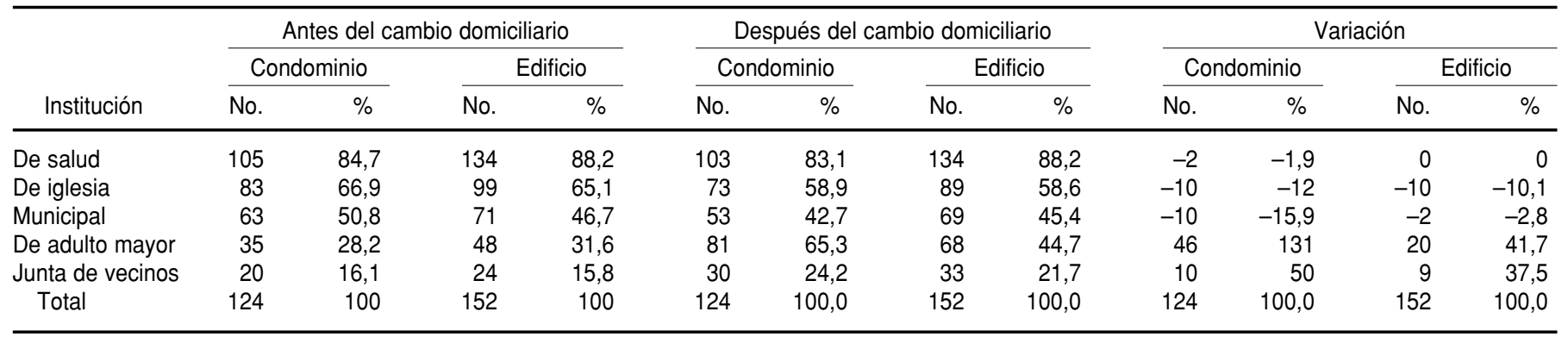

mación): 6,4\% y, finalmente, la de tipo instrumental (cuidado, transporte, curaciones, labores de hogar): 5,1\%.

En cuanto a la ayuda específica, se observa que los ítems "pensión" y "remedios" son los más mencionados: 17,7\% y $13 \%$, respectivamente. A continuación aparecen las ayudas emocionales como "compañía", "escucha", "afecto" y "consuelo". Sólo en tres categorías se observan diferencias significativas entre los AM de los dos tipos de viviendas: "cuidado" $\left(\chi^{2}=7,881 ; P=0,005\right)$, "labores de hogar" $\left(\chi^{2}=11,169 ; P=0,001\right) \mathrm{y}$ "comida" $\left(\chi^{2}=6,907 ; P=0,009\right)$, siendo las dos primeras categorías mayores en condominio. Por el contrario, el ítem "comida" resultó mayor para los AM en edificio, sin tener un fundamento claro para esta diferencia. De estos tres casos, sólo el ítem "comida" cuenta con un porcentaje importante del total de respuestas $(25,4 \%)$.

Frecuencia de ayuda recibida. $\mathrm{Al}$ analizar los resultados según frecuencia y tipo de ayuda (emocional, material, instrumental, cognitiva), se puede señalar que ambos grupos de AM reciben la ayuda material sobre todo en forma mensual y la ayuda emocional de manera marcadamente semanal (74\% y $82 \%$, respectivamente). En la categoría cognitiva "información para resolver problemas" se encontraron diferencias porcentuales entre ambos grupos de AM: en los condominios, la frecuencia diaria es la destacada, en tanto que en los edificios la frecuencia es, en su mayoría, semanal. Por otra parte, en ninguno de los grupos se concentra la ayuda instrumental en algún periodo: en los condominios se distribuye igualitariamente en forma semanal y diaria $(32 \%)$ y en los edificios es, sobre todo, mensual (52\%) y después semanal (30\%).

Ayuda que los AM otorgan a otros. En el caso de los condominios, $44 \%(n=55)$ de los AM ayudan a otras personas; en los edificios, esa cifra desciende a $26 \%$ $(n=39)$; esta diferencia es estadísticamente significativa: $\chi^{2}=9,845 ; P=0,002$. De los AM en condominios, $14,5 \%$ ayudan sobre todo a los hijos, en tanto que sólo lo hacen $7 \%$ de los que residen en edificios, con una diferencia estadísticamente significativa: $\chi^{2}=8,143 ; P=0,004$. Asimismo es importante la diferencia de ayuda con los vecinos: en condominios representan $12 \%$ de los beneficiados; en edificios $9,2 \%$.

\section{DISCUSIÓN}

Los AM beneficiados por el PVBAM han experimentado un cambio drástico en su entorno al mudarse de vivienda y de barrio. Esto los lleva a percibir lo que les rodea de manera diferente, tanto desde su mirada al contexto de la vivienda y el barrio, como en cuanto al sentido de inclusión y exclusión social con la nueva comunidad (10).

Redes con personas. A la luz de los resultados se hace evidente que el único cambio significativo ocurrió en la relación con personas fuera de la familia, que en el caso de los AM en edificio ha significado el aumento en el número de personas de su red social. Lo anterior se explica porque residen junto a muchas otras familias con las que deben tener algún grado de relación, aun cuando sus intereses puedan ser distintos. Por otro lado, también se observa que el vínculo padres-hijos es el más fuerte, y que se mantuvo inalterable a pesar del tipo de vivienda o barrio al cual fueron trasladados los AM. Las restantes relaciones mostraron disminución en ambos grupos de AM, aun cuando no fueron estadísticamente significativas. La principal causa de esta disminución fue la distancia, misma que deberá considerarse en futuros programas similares al estudiado.

Redes con organizaciones o instituciones. Es evidente que no hay cambios sustanciales o importantes con las instituciones de salud, dado que estos AM necesitan considerablemente el apoyo sanitario. Sin embargo, en lo que respecta a los nexos con otras instituciones como son las de iglesias y las municipalidades, al no ser tan determinantes en las soluciones más básicas e inmediatas, los vínculos antiguos se pierden y se requieren mayores esfuerzos para restablecerlos.

En los dos grupos de AM, la mayor vinculación con los centros de AM puede reflejar la búsqueda de ayuda cuando han disminuido otras redes. Esta relación es de mayor magnitud, estadísticamente significativa en el caso de los condominios, lo cual puede deberse a que estos AM viven en comunidad o tienen intereses y necesidades comunes, lo que facilita la acción en conjunto. El hecho de que vivan en comunidad permite que las autorida- 
des les ofrezcan planes para realizar actividades comunes. Los AM en condominio logran generar un sentido de comunidad con sus pares, lo que facilita su interacción. Los aprendizajes se potencian cuando son compartidos para tratar de solucionar un problema común (11). En cambio, la situación de los AM en edificio es diferente debido a que se encontraban aislados, insertos en un contexto de familias en diferentes etapas del ciclo vital familiar $y$, por lo tanto, con intereses distintos.

Las instituciones son redes de apoyo importante para el AM, y sería de interés evaluar constantemente su interacción con ellos; las personas tienen más posibilidades de salir adelante y superar los problemas gracias a este tipo de redes (12).

Tipo de ayuda. Los AM reciben diferentes tipos de ayuda, entre los que destacan la material, en primer lugar, seguida por las de carácter emocional. Cabe hacer notar que el rol del consultorio es más relevante en el caso de los AM en condominios, lo cual podría explicarse porque al estar juntos tienen mayor acceso a información y apoyo correspondiente a su grupo etario, con lo cual logran una sinergia positiva para satisfacer sus necesidades de salud (vacunas, atención de morbilidad crónica y aguda, entrega de fármacos, recepción de suplementos alimenticios, etcétera). En cambio los AM en edificio se encuentran aislados entre sí, en un contexto donde las personas no necesariamente comparten sus necesidades de salud.
Ayuda a otros. No obstante las carencias que presentan los AM estudiados, prácticamente la mitad deben ayudar a miembros de su familia y a amigos. Tradicionalmente se piensa que los AM dependen de las generaciones más jóvenes $\mathrm{y}$, en general, este enfoque no considera que en muchas ocasiones los AM desempeñan un papel activo en la provisión de servicios y recursos financieros a favor de las generaciones más jóvenes (13). Llama la atención que no obstante la precariedad de su situación económica, muchos AM prestan ayuda material (alimentación, calefacción) a otras personas. La mayor ayuda otorgada en condominio, en comparación con lo que sucede en los edificios, se debe a distintas causas; una de ellas puede ser la mayor vecindad con personas que se encuentran en la misma condición de vulnerabilidad, lo que favorece la solidaridad. Empero, debería ser un motivo de atención la ayuda en dinero que puedan prestar, ya que la explotación financiera es uno de los maltratos que sufren las personas de edad avanzada (14).

\section{Comentarios generales}

Pese a las pérdidas de redes que se producen, las personas consideraron como positivo su traslado, y ambos grupos estaban satisfechos ya que su nueva vivienda es de mejor calidad que la anterior. Los AM en condominio manifestaron mayor satisfacción que los de edificio porque se sienten más protegidos en su entorno del barrio. El concepto de calidad de vida se debe valorar desde la perspectiva de las personas y no de los evaluadores (5).

Es relevante generar un programa de apoyo al realizar estos traslados, dado que durante el proceso deben evaluarse las necesidades que surgen y corregir errores en la inserción de AM a nuevos barrios (15). Es necesario promover la creación de nuevos vínculos con instituciones formales como el consultorio, el municipio y la iglesia, así como con otras organizaciones comunitarias como son las juntas de vecinos, los centro de madres, etcétera. Esto facilitaría el reconocimiento de los AM, su acogida, protección y eventual solidaridad con ellos. Es importante que la sociedad valore a los AM, considerando que son personas que han llegado a una etapa de mayor madurez y que han adquirido sabiduría (16).

Dada la vulnerabilidad en aumento del AM, los programas de vivienda social tendrían que ser evaluados periódicamente considerando los distintos ámbitos analizados (17). La sociedad debe colaborar con las generaciones mayores demostrando una "madurez filial" que la lleve a asumir sus responsabilidades hacia ellos (18). No se debe olvidar que el protagonismo lo tienen ellos, y que debe estimulárseles para conformar comunidades y asumir un rol activo en su interacción con los demás actores sociales, especialmente con los sectores de salud, seguridad ciudadana, etcétera (19).

\section{REFERENCIAS}

1. Rodríguez A, Rodríguez E. Antropología y cultura médica contemporánea. Loja: Universidad Técnica Particular de Loja; 2004.

2. Del Popolo. Características sociodemográficas y socioeconómicas de las personas de edad en América Latina. Santiago: CEPAL/CELADE; 2001.

3. Cuevas M. El doble yugo de la pobreza en la vejez. El Mercurio 200421 de noviembre. Global Action on Aging (GAA). [Sitio en Internet] Hallado en: http://www.globalaging.org/ elderrights/world/2004/yugo.htm. Acceso el 10 de noviembre 2006

4. Albrecht R, Morales J. ¿Por qué envejecemos de manera diversa? En: Rodríguez R, Morales J, Encinas J, Trujillo Z, D`Hyver C, eds. Geriatría. México: McGraw-Hill Interamericana; 1999. Pp.29-31.

5. Rodríguez R, Mendoza L. Calidad de vida. En: Rodríguez R, Morales J, Encinas J, Trujillo Z, D'Hyver C, eds. Geriatría. México: McGraw-Hill Interamericana; 1999. Pp.297-9.
6. Comisión Económica para América Latina y el Caribe/Centro Latinoamericano de Demografía. Reunión de Expertos en Redes de Apoyo Social de las Personas Mayores: el rol del Estado, la familia y la comunidad. Santiago: CELADE; 2003.

7. Murad P. Transferencias informales de apoyo de los adultos mayores en América Latina y el Caribe; estudio comparativo de encuestas SABE. En: Notas de población. Santiago, Chile: CEPAL/CELADE; 2003. Pp. 175-218. 
8. Chadi M. Redes sociales en el trabajo social. Buenos Aires: Editorial Espacio; 2000.

9. Guzmán JM, Huenchuan S, Montes de Oca V. Redes de apoyo social de las personas mayores: Marco conceptual. En: Notas de población. Santiago, Chile: CEPAL/CELADE; 2003. Pp. 35-70.

10. Morandé P. Comportamientos sociales en la ciudad. En: Tupper P, ed. Hacer ciudad. Santiago: Centro Chileno de Urbanismo; 2004. Pp. 39-48.

11. Dabas E. Red de redes. Buenos Aires: Editorial Paidos; 2001.

12. Aylwin N, Solar M. Trabajo social familiar. Santiago: Ediciones Universidad Católica de Chile; 2002.
13. Aranibar P. Acercamiento conceptual a la situación del adulto mayor en América Latina. Santiago: CEPAL/CELADE; 2001.

14. Bitondo C, Kim L. Maltrato en el adulto mayor: abuso, negligencia y explotación. En: Landefeld C, Palmer R, Jonson M, Johston C, Lyons W. Diagnóstico y tratamiento en geriatría. México: El Manual Moderno; 2005. Pp. 425-30.

15. Pérez G. Elaboración de proyectos sociales. Madrid: Narscea, S.A. Ediciones; 2002.

16. Díaz C. Pedagogía de la ética social. México: Editorial Trillas; 2004.

17. Capp M. Temas legales y éticos comunes. En: Landefeld C, Palmer R, Jonson M, Johston C, Lyons W. Diagnóstico y tratamiento en ge- riatría. México: El Manual Moderno; 2005. Pp 491-8.

18. Mishara B, Riedel R. El proceso de envejecimiento. Madrid: Ediciones Morata S. L.; 2000.

19. Krauss A, Marín P, Barros C, Peláes M, Mardonez F, Oyarso C, et al. Reto a la independencia del adulto mayor. Santiago: Pontificia Universidad Católica de Chile. Programa para el Adulto Mayor; 2001

Manuscrito recibido el 2 de marzo de 2007. Aceptado para publicación, tras revisión, el 20 de diciembre de 2007.

ABSTRACT Objectives. To identify, compare, and analyze the social networks of two groups of poor, older adults in two types of public housing projects in Chile, in terms of the types of relationships and the frequency and purpose of their interactions, before and

\section{Changes in the social networks of older adults who are beneficiaries of public housing projects in Chile} after moving to their new homes.

Methods. A descriptive study was conducted of public housing beneficiaries in apartment buildings versus condominiums. The study pool was the group of people assigned to housing in buildings $(n=152)$ and condominiums $(n=124)$ from 1998 to 2001. A questionnaire was administered in their homes.

Results. Most study participants indicated that the move to public housing meant leaving their old neighborhood for a new one, a change that, among other consequences, produced changes in their social networks. In general, both groups have similar social networks, with their children being the strongest source of support, both before and after the move. After the move, social interaction generally decreased, except among the adults living in apartment buildings, for whom it increased slightly with family members, and in a superficial manner, with others who had weak connections and were neither friends nor family. Interactions with establishments also decreased, although interactions with senior services increased for the condo residents from $28 \%$ to $65 \%$ and for apartment residents from $31 \%$ to $45 \%$.

Conclusions. It is recommended that similar programs strive to preserve and strengthen the social networks in place prior to moving and encourage the formation of relationships in the new residences.

Key words Aged, public housing, housing for the elderly, poverty, Chile. 